\title{
FIBER OPTIC SENSOR FOR THE MEASUREMENT OF CONCENTRATION AND REFRACTIVE INDEX OF LIQUIDS BASED ON INTENSITY MODULATION
}

\author{
A.L. CHAUDHARI \\ Department of Electronics, Arts, Science \& Commerce College, CHOPDA \\ Dist. Jalgaon PIN 425107 India. \\ E-mail: chaudharial@yahoo.co.uk \\ A. D. SHALIGRAM \\ Department of Electronic Science, University of Pune, PUNE-411 007 India.
}

\begin{abstract}
This paper reports the use of fiber optic sensor consists of two-fiber probe and reflector for the measurement of concentration and refractive index of liquids based on the reflective intensity modulation. Conventionally such a sensor is used for measuring micro-displacement of fiber tip with reference to the reflector. In one of the previously reported work we have established that if the separation between fiber tips and reflector is maintained constant the same arrangement can be directly used for sensing refractive index changes of medium. The liquid under test fills the gap between probe and reflector. Depending upon the refractive index of liquid, angle of emittance will change which will decide the received output power by receiving fiber. Apart from pure solvents which have different refractive indices, there are mixtures of various types which also alter the refractive index of a given medium. The developed sensor is used to detect the concentration of the alcohols like methanol \& ethylene glycol in mixture with water.
\end{abstract}

Keywords: Fiber optic sensor, intensity modulation, refractive index, alcohols.

\section{Introduction}

Many advances have been made in recent years in the use of fiber optic sensors(FOSs). The advantages of FOSs are well known which include high sensitivity, EMI, spark free, minimal intrusiveness due to their relatively small size and light weight. Even though a range of FOSs are reported in the literature, intensity based optical fiber sensors represent one of the earliest and perhaps most basic type of optical fiber sensor [1].

Refractive index is an important characteristic of optical material. Simple and highprecision method of measuring refractivity of liquid has been required for the material analysis and also for applications like environmental pollution monitoring of both river and sea water[2]. 
Precise measurement of refractive index of transparent liquids is vital to design of optical instruments and is also of great value in chemical work. The knowledge of refractive index of a substance is useful in identifying and determining the concentration of organic substances.

Methanol $(\mathrm{CH} 3 \mathrm{OH})$ is the simplest alcohol, containing one carbon atom. It is a colorless, tasteless liquid with a very faint odor and is commonly known as "wood alcohol." Methanol is used in a number of consumer products, including paint strippers, duplicator fluid, model airplane fuel, and dry gas. Most windshield washer fluids are 50 percent methanol. Methanol's physical and chemical characteristics result in several inherent advantages as an automotive fuel. The commercialization of methanol-powered fuel cells will offer practical, affordable, long-range electrically-powered vehicles with zero or near-zero emissions while retaining the convenience of a liquid fuel.

Ethylene glycol is a clear, colorless, odorless, viscous liquid with a sweet taste, that can produce dramatic toxicity. It is commonly found in homes and industry. It is found most commonly in antifreeze, automotive cooling systems, and hydraulic brake fluids. In an industrial setting it is used as a solvent or as the raw material for a variety of processes[6]. Many cases of ethylene glycol poisoning results from accidental ingestion by children who can take in large amounts since the substance tastes good. Alcoholics may also ingest this substance as an ethanol substitute.

Considering the important uses of these alcohols, it is necessary to detect the concentrations of these alcohols in mixture with water. The developed sensor is used to detect the concentration.

\section{Experimentation}

Different concentration methanol solutions were prepared by mixing pure methanol with distilled water. Range of concentration is from $0-100 \%$. The developed sensor was used to detect the concentration of methanol in mixture. Ethylene glycol is not easily soluble in water. So magnetic stirring was done to get homogenous mixture. Magnetic stirring was carried out for 5 minutes at room temperature. Rest of the procedure is same as explained above. Developed fiber optic sensor consists of fiber probe and reflector based on intensity modulation is used for measurement of concentration and refractive index using these solutions[1].

\section{Results and Discussion}

The response of developed sensor for methanol water mixture with different colored LED illuminations is shown in figures 1(a) and figure 1(b). 


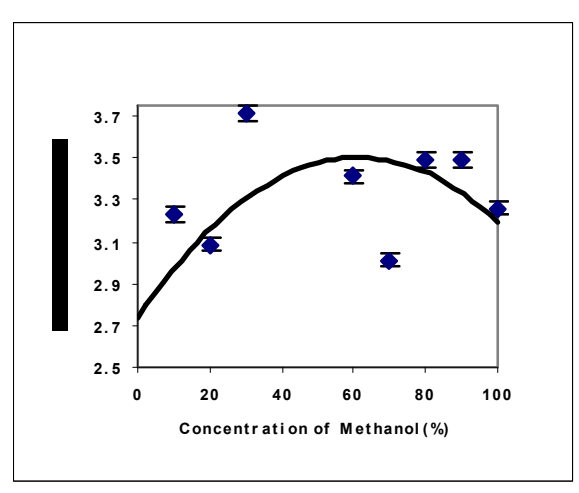

Figure 1(a) Response of FO sensor for Methanol-Water mixture for RED LED

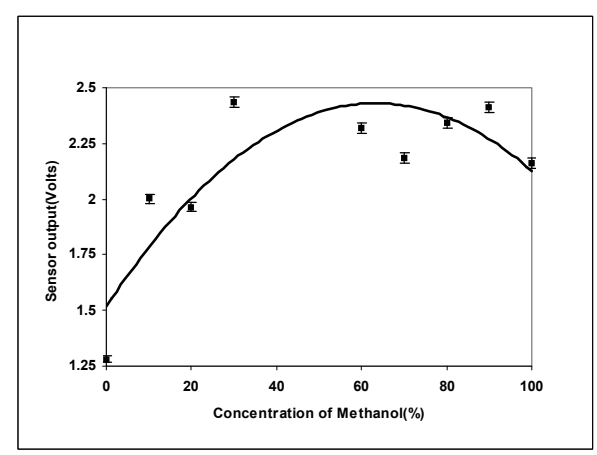

Figure 1(b) Response of FO sensor for Methanol-Water mixture for BLUE LED

It is seen that as methanol concentration in water increases the sensor output initially increases, goes through maxima and reduces again. Literature survey revealed that indeed such behavior of refractive index variation is reported. Figure 2 shows the experimental observation obtained by Jon P. Longtin and Ching-Hua Fan[3] along with the standard data obtained from CRC Handbook4]. Longtin and Fan have carried out the measurement with the help of a Laser based refractive index measurement system with a UDT SL5-2 linear position sensor and PC based system to precisely monitor the beam deflection through cuvette. The similarities between the nature of curves from figures 1 and 2 are clearly observable. The deviations of the observed sensor output voltages from the trend curves in figure 1 can be attributed to the experimental approximations in preparing the mixtures. However, the purpose of demonstrating the usability of developed sensor is served. Figure 1 also shows the color depend ace of the variation.

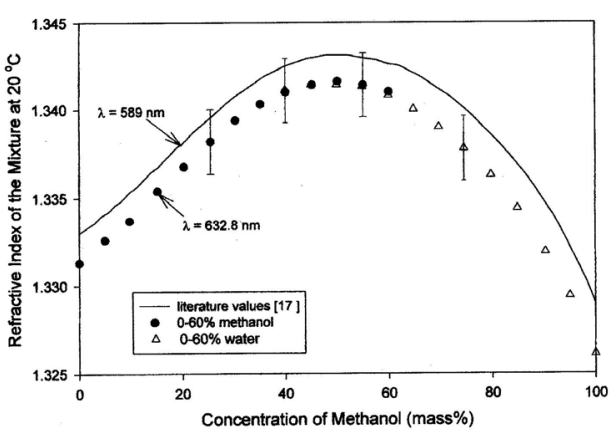

Figure 2 Methanol-water mixture refractive index measurement

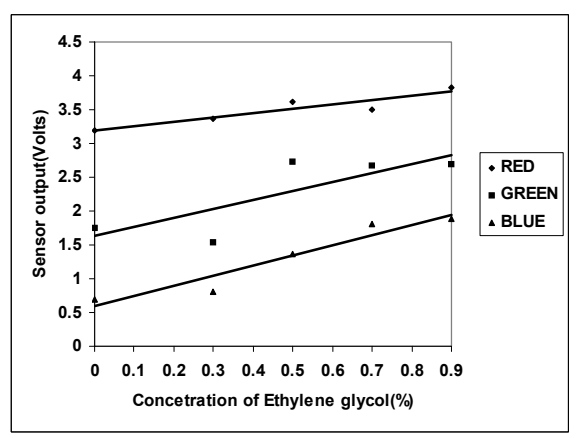

Figure 3 Response of FO sensor for Ethylene glycol-Water mixture 


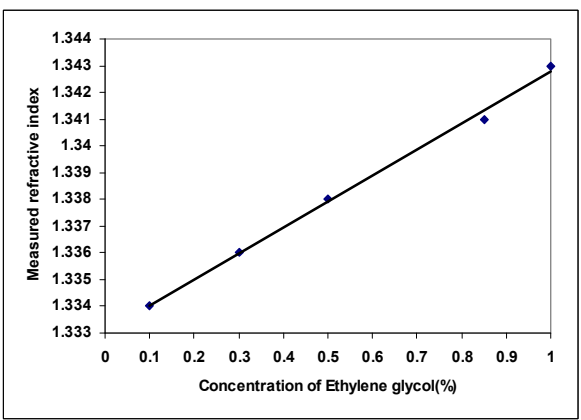

Figure 4 Variation of refractive index for Ethylene glycol-Water mixture.

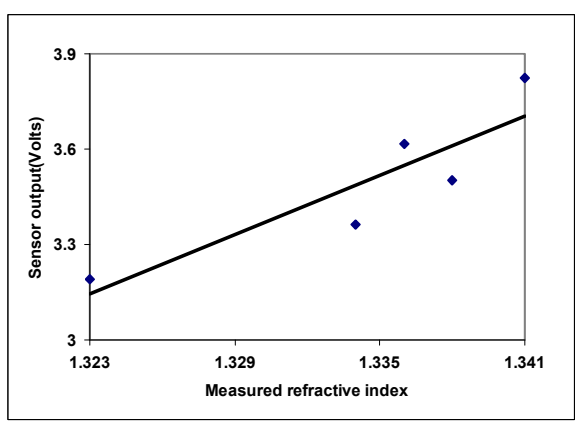

Figure 5 Response of FO Sensor for variation in refractive index

Figure 3 shows the results obtained for the ethylene glycol-water mixture. There is a almost linear rise in the sensor output predicting a linear change in the refractive index of mixture as the ethylene glycol concentration increases. Refractive indices of the same samples were measured using standard Abbe's refractometer. Figure 4 shows the variation of refractive index of the mixture with concentration. Similarity of the nature of two plots is revealing.

Figure 5 shows the sensor output voltages plotted against the measured refractive index. Esteban et al[5] have used a surface plasmon resonance based fiber optic sensor for the determination of refractive index of ethylene glycol and obtained similar results. The empirical formula for the refractive index variation of ethylene glycol-water mixture with concentration is as[6]

$$
n(T)=n_{\mathrm{H}_{2} \mathrm{O}}(T)+0.111 \frac{V_{e t h}}{V_{\text {tot }}}
$$

Where $n(T)=$ refractive index of mixture, $n_{\mathrm{H} 2 \mathrm{O}}(T)=$ refractive index of water

$V_{\text {eth }}=$ volume of ethylene glycol \& $V_{\text {tot }}=$ total volume of mixture

This also suggest linear variation further supporting the results shown in figure 5 .

\section{Conclusion}

Refractive index is an important characteristics of optical materials. In this paper the use of fiber optic sensor for refractive index variation measurements has been tested using alcohol water mixtures. The mixtures used are methanol-water and ethylene glycol-water to get a change in refractive index. Result $\mathrm{s}$ obtained are matching with the reported results in literatures. The developed sensor bears a good promise. With appropriate mechanical design and circuit packaging the developed sensors can be used in many fields like chemical industries, food industries, sugar factory. 


\section{Acknowledgement}

Author(ALC) wishes to thanks the Dr. Suresh G. Patil, Founder President, Adv. Sandeep Suresh Patil, President, Adv. V. D. Joshi, Vice President, Dr. Smita S. Patil, Secretary, MGSM, Chopda, Dr. D. D Patil, Principal, Faculty of Electronics \& Physics Department, Arts, Science \& Commerce College, Chopda Dist. Jalgaon, Maharashtra India for giving the cooperation during the research work.

\section{References}

1. A. L. Chaudhari and A. D. Shaligram, Sensors and Actuators A 100(2002), pp160-164.

2. Hiroyuki Hattori, Hirotogu Yamanaka, Hendrik Kurniawan, Sadathiro Yokoi, and Kiichiro Kagawa, Applied optics, 36(22)(1997),5552-56.

3. Jon P. Longtin and Chin_Hua Fan, "Precision Laser Based Concentration and Refractive Index Measurement of Liquids", icroscale Thermophysical Engineering, 2(1998), pp261-272.

4. D. R. Lide, in D. R. Lide(ed.), CRC Handbook of Chemistry and Physics, CRC Press, Boca Raton, FL, 1998.

5. Oscar Esteban, Maria Cruz-Navarrete, Augstin Gonzalez-Cano, and Eusebio Bernabeu, Applied Optics, 38(25)(1999), pp52675271.

6. R. Alonso, "Etudio teorico y experimental de dispositivos opticos basados en el acoplamiento entre el modo guiado poruna fibra optica y estructuras multicapa incluyendo medios metalicos", Ph. D. dissertation, Uinversity of Zaragoza, Spain, 1995. 